Short Communication

\title{
Accelerated azo dye removal by biocathode formation in single-chamber biocatalyzed electrolysis systems
}

\author{
You-Zhao Wang ${ }^{\mathrm{a}}$, Ai-Jie Wang a,b,*, Wen-Zong Liu ${ }^{\mathrm{b}}$, De-Yong Kong ${ }^{\mathrm{a}}$, Wen-Bo Tan ${ }^{\mathrm{a}}$, Chong Liu ${ }^{\mathrm{a}}$ \\ a State Key Laboratory of Urban Water Resource and Environment, Harbin Institute of Technology, No. 73 Huanghe Road, Harbin 150090, PR China \\ ${ }^{\mathrm{b}}$ Research Center for Eco-Environmental Sciences, Chinese Academy of Sciences, Beijing 100085, PR China
}

\section{H I G H L I G H T S}

- Biocathode could form in single-chamber BES by absorbing the electrochemical activity microbes from anode biofilm naturally. - From abiocathode to biocathode, both the polarization current and charge transfer resistance were improved in 2 weeks.

- Azo dye removal was accelerated in biocatalyzed electrolysis system with biocathode comparing with abiocathode.

\section{A R T I C L E I N F O}

\section{Article history:}

Received 7 May 2013

Received in revised form 11 July 2013

Accepted 13 July 2013

Available online 24 July 2013

\section{Keywords:}

Biocatalyzed electrolysis system

Biocathode

Azo dye removal

\begin{abstract}
A B S T R A C T
Biocatalyzed electrolysis systems (BES) have been the topic of a great deal of research. However, the biocathodes formed in single-chamber BES without extra inocula have not previously been researched. Along with the formation of biocathodes, the polarization current increased to $1.76 \mathrm{~mA}$ from $0.35 \mathrm{~mA}$ of abio-cathodes at $-1.2 \mathrm{~V}$ (vs. SCE). Electrochemical impedance spectroscopy (EIS) results also indicated that the charge transfer resistance $\left(R_{\mathrm{ct}}\right)$ was decreased to $148.9 \Omega$, less than $1978 \Omega$ of the abio-cathodes cleared. The performance of the biocathodes was tested for azo dye decolorization, and the dye removal efficiency was $13.3 \pm 3.2 \%$ higher than abio-cathodes with a $0.5 \mathrm{~V}$ direct current (DC) power supply. These aspects demonstrate that biocathode accelerates the rate of electrode reaction in BES and comparing with noble metal catalysts, biocathodes have low toxicity or non-toxic and reproducible properties, which can be widely applied in bioelectrochemical field in the future.
\end{abstract}

(c) 2013 Elsevier Ltd. All rights reserved.

\section{Introduction}

In traditional bioelectrochemical systems (Logan et al., 2006), the electric energy is generated from chemical energy stored in organic matter (microbial fuel cells, MFC). Even today, most related research focuses on the MFC material and energy generation. However, biocatalyzed electrolysis systems (BES) can convert the organic matter from anodes to electrons, which can be transported to $\mathrm{CO}_{2}, \mathrm{H}^{+}$and other acceptors to generate $\mathrm{CH}_{4}, \mathrm{H}_{2}$ in the cathodes with little DC power supply (Liu et al., 2008), thus saving a significant amount of electrical energy compared to the pure electrochemical method. This discovery extends the application range of BES, even allowing for removal of refractory pollutants by cathode reduction (Huang et al., 2010; Shen et al., 2012; Wang et al., 2011b). Azo dye wastewater could be also treated by cathodes, since azo dye can accept electrons from cathodes which will break azo bonds (Mu et al., 2009). An additional DC supply is necessary

\footnotetext{
* Corresponding author at: State Key Laboratory of Urban Water Resource and Environment, Harbin Institute of Technology, No. 73 Huanghe Road, Harbin 150090, PR China. Tel.: +86 4586282195.

E-mail address: waj0578@hit.edu.cn (A.-J. Wang).
}

since the electrochemical reduction needs to overcome a lower potential. However, BES treatment of wastewater still faces many challenges, such as cathode catalysis. Platinum is as effective cathode catalyst often used in Laboratory BES. But the expensive price and lack of long-term sustainability increases construction cost, making it unsuitable for practical, large-scale engineering applications. Biocathodes with electrochemical activity microbes are a good substitute for platinum, due to their effective performance as catalysts (Carbajosa et al., 2010; Erable et al., 2010).

In BES, bioanodes transport electrons through electrochemical activity microbes that attach to the anode surface (Jeremiasse et al., 2009). These important microbes may be transported to cathodes for transporting electrons to electron acceptors in single-chamber BES without ion exchange membranes. The primary goal of this study was to investigate whether biocathodes can be formed in single-chamber BES automatically, naturally selected from anode biofilm without extra inocula. The second objective was to measure improvement on catalysis efficiency for azo dye removal when biocathodes formed, as opposed to abiocathodes. Electrochemical monitoring methods were performed for evaluating its property in biocathode formation. 


\section{Methods}

\subsection{BES set-up}

Three identical laboratory scale BES were constructed to be cuboid (volume $140 \mathrm{ml}$ ) out of Plexiglas. Both anodes and cathodes are carbon brush (ID $4.5 \mathrm{~cm} \times L 4.0 \mathrm{~cm}$ ) and a saturated calomel electrode (SCE) reference electrode was placed in the BES (Wang et al., 2013). The DC power supply between the anode and cathode was $0.5 \mathrm{~V}$. The bioanode was incubated in a MFC with acetate as the carbon resource in our laboratory (Wang et al., 2011a). The BES was operated by batch mode at room temperature $23 \pm 2{ }^{\circ} \mathrm{C}$ and the solution was replaced every 7 days. The composition of the artificial azo dye wastewater was as follows $(\mathrm{g} / \mathrm{L})$ : amido Black10B, 0.1; acetate, 0.82; $\mathrm{Na}_{2} \mathrm{HPO}_{4} \cdot 12 \mathrm{H}_{2} \mathrm{O}, 11.55 ; \mathrm{NaH}_{2} \mathrm{PO}_{4} \cdot 2 \mathrm{H}_{2}$ $\mathrm{O}, 2.77 ; \mathrm{NH}_{4} \mathrm{Cl}, 0.41 ; \mathrm{KCl}, 0.14$. The reaction solution only needed to pump nitrogen for 15 min to clear the oxygen, followed by high temperature sterilization.

\subsection{Analysis of reactor performance}

The current was monitored every 15 min by measuring the resistor voltage with a voltage collection instrument. To examine the cathode, at the start of every cycle two electrochemical methods were used: cyclic voltammetry was performed from 0.4 to $-1.2 \mathrm{~V}$ (vs. SCE) with a scan rate of $10 \mathrm{mV} / \mathrm{s}$; the EIS method was used to characterize the $R_{\mathrm{ct}}$ of cathodes (3-electrode mode), frequency range was taken from 100 to $10 \mathrm{mHz}$ using a $10 \mathrm{mV}$ sine wave and the data was simulated by ZsimpWin3.10 software. After biocathode formation ( 3 times solution replacements), the azo dye removal efficiency was measured for $10 \mathrm{~h}$. However, previous studies (Hong et al., 2012) show that azo dye can be removed at anodes in MFC in an anaerobic role. In order to avoid the anode influencing the removal efficiency and only show the changes in cathode, ion exchange membrane (IEM) was used to separate both the anode and cathode tentatively. The amido black 10B concentration was measured by spectrophotometry and its maximum absorption wavelength was $618 \mathrm{~nm}$. The azo dye removal efficiency was evaluated as follows:

Removal efficiency $(\%)=\left(1-C_{t} / C_{0}\right) \times 100 \%$

where $C_{0}$ is the initial concentration of amido black $10 \mathrm{~B}$, and $C_{t}$ is the concentration at reaction time $t$.

\section{Results and discussion}

\subsection{Biocathode formation in single-chamber BES}

At the start of the experiments, the single-chamber BES with fresh abiotic cathode was set up by using a $0.5 \mathrm{~V}$ DC power supply. After replacement of the solution, the anode and cathode potentials achieved a stable -0.5 and $-1.0 \mathrm{~V}$ (vs. SCE) respectively (Fig. 1A). After 2 weeks, the current of BES showed a great change: at the initial time, the current was about $0.2 \mathrm{~mA}$, which increased to $0.4 \mathrm{~mA} 7$ days later, until achieving a maximal current of $0.67 \mathrm{~mA}$ after 14 days (Fig. 1B). This result showed a beneficial effect of BES, since larger a current indicates that the cathodic reduction was accelerated. When the anode was retaining a stable status over a long time, the source of the changes was only attributed to what occurred on the cathodic surface.

To examine what happened in the cathode, SEM analysis was used to observe the surface of the cathode carbon brush. On biocathode, the bacteria attached to the carbon fiber and gathered into clusters, comparing with abio-cathode cleared (Supplementary data). Similar SEM images are also found in previous biocathode studies (Erable et al., 2010). To further confirm the biocathode formation, the electrochemical monitoring method was performed in three different stages. As seen from Fig. 2A, the CV revealed significant changes. No redox peak was observed from the cathode in any of the three different stages, but the polarization currents changed distinctly. At potential $-1.2 \mathrm{~V}$ (vs. SCE), the polarization current at 14 days increased to 1.76 from 0.35 of the initial time. The absence of any redox peaks can possibly be attributed to the insufficient sensitivity of the cathode carbon brush, or insufficient coverage of microbial biomass.

EIS method was used in biocathode formation as shown in Fig. 2B. The semicircles corresponded with $R_{\mathrm{ct}}$ in the cathode interface. As time went on, the semicircle was decreased gradually, meaning a decrease in the $R_{\mathrm{ct}}$ and benefit to electrochemical reaction, such as hydrogen reduction, azo bonds breaking, etc. After simulating an equivalent circuit (He and Mansfeld, 2009), the value of $R_{\mathrm{ct}}$ was found to be 607.7 and $148.9 \Omega$ at 7 and 14 days respectively, comparing with a fresh cathode of $1978 \Omega$ the $R_{\mathrm{ct}}$ decreased 13 times over time. From both CV and EIS, it is obvious that the biocathode increased the reaction current and accelerated the rate of electrode reaction.

As demonstrated above, biocathodes can be formed in singlechamber BES automatically, this finding is conducive to biocathode formation because it uses efficient inocula as opposed to sludge, sediment, etc., which may contain useless microbes (no ability of extracellular transfer electron), that will increase electrochemical reaction resistance and result in wasting available electrode surface. In this work, cathode inocula from anode biofilm, which has already had ability of extracellular transfer electrons. Comparing natural inocula, anode biofilm has gathered lots of electrochemical activity microbes which transfer electron to electrode. This ability
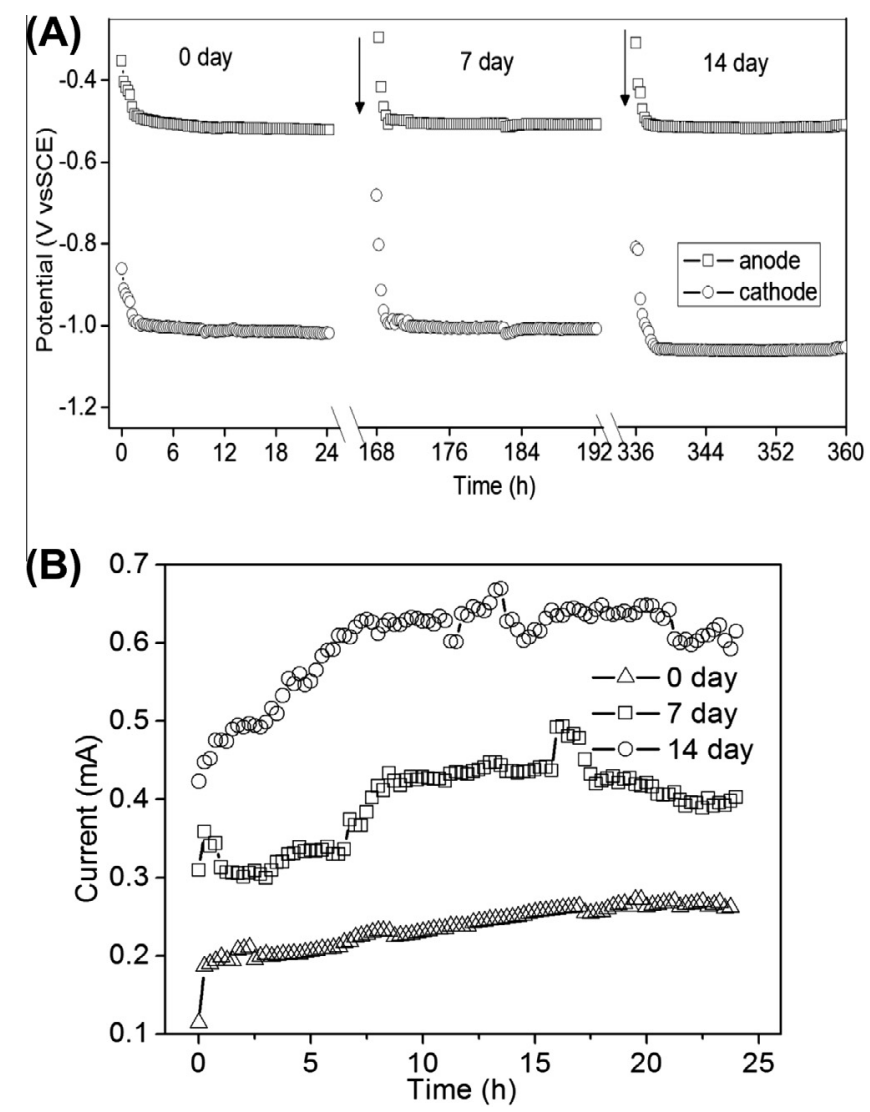

Fig. 1. Changes in single-chamber BES in 14 days, (A) potential evolution (the arrows indicate when replace solution) and (B) current evolution. 
can convert form electrode to microbes themself in previous study (Strycharz et al., 2008). In other words, between electrode and microbes, these microbes not only can donate the electrons to anode which served as an electron acceptor, but also accepter electrons form electrode which poised a low potential.

\subsection{Azo dye removal by biocathode}

After formation of the biocathode, its capability in azo dye removal was tested. It is well known that a possible result of azo bonds accepting electrons is decolorization of the cathode. Of course, IEM is an expensive material that is unsuitable for largescale engineering, but in laboratorial BES it is highly effective for researching cathode changes without disturbing the anode.

When artificial azo dye wastewater was injected into the cathode chamber, as shown in Fig. 3A, the wastewater became colorless in a short time. Azo dye removal efficiency and BES current are shown in Fig. 3B, the removal efficiency increased along with DC power supply. At $0.5 \mathrm{~V}$ DC (cathode potential $-1.0 \mathrm{~V}$ vs. SCE), the removal efficiency of the biocathode achieved $81.7 \%$ within $10 \mathrm{~h}$, an increase of $13.3 \%$ compared to the abio-cathode. Similar results were obtained from current acquisition, the current with biocathode achieved $0.45 \mathrm{~mA}$, twice that of the abio-cathode.

On the other hand, this difference was inconspicuous with decreasing DC voltage until reaching $0 \mathrm{~V}$. This might be caused by microbes which need lower potential to transport electrons to azo dye from the cathodic surface. The $0 \mathrm{~V} \mathrm{DC}$ power supply
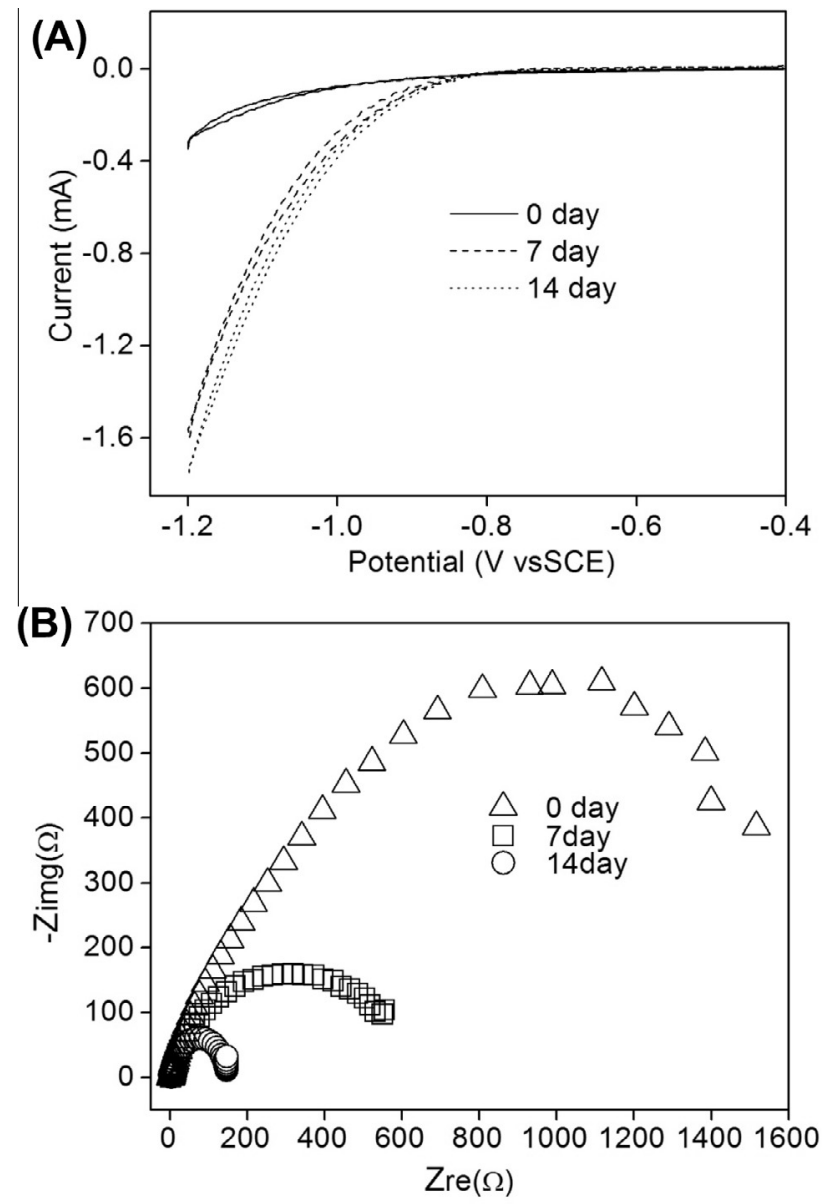

Fig. 2. Electrochemical monitoring in biocathode formation in third stage (A) CV results; (B) EIS results. provide a $-0.5 \mathrm{~V}$ (vS. SCE) cathode potential, which cannot accelerate the extracellular electron transfer lead to the current and azo dye removal efficiency were similar with abio-cathode. When the cathode potential overcame the limit, the electrons can be taken from electrode surface faster, and then joined to azo bond breaking by azo reductase which exists in most of microbes. However, all aspects of the experiment demonstrate that the biocathode can accelerate the azo dye decolorization in a low potential. This bio-electrode has a potential advantage for bioremediation of azo dye industrial wastewater in condition where electron donor is deficient and electrode can be used as electron donor. Compared to the noble metal catalyst, biocathodes have a low toxicity or non-toxic and reproducible property, and have a wide range of practical applications in the future bioelectrochemical field.

\section{Conclusions}

This work has demonstrated that biocathodes can automatically and naturally form in single-chamber BES by absorbing the electrochemical activity microbes from bioanode. Both the polarization current and $R_{\mathrm{ct}}$ were improved. For evaluation using amido black $10 \mathrm{~B}$ as the electron acceptor, its removal efficiency was raised $81.7 \%$ than that of the abiocathode within $10 \mathrm{~h}$. This cathodic biofilm was conducive to catalyzing pollutant reduction as a replacement of expensive catalysts, and is thus beneficial for practical application in the future. Further, effective strains can be isolated from the biocathode, allowing more efficiency and perhaps new discoveries for future research on biocathodes.
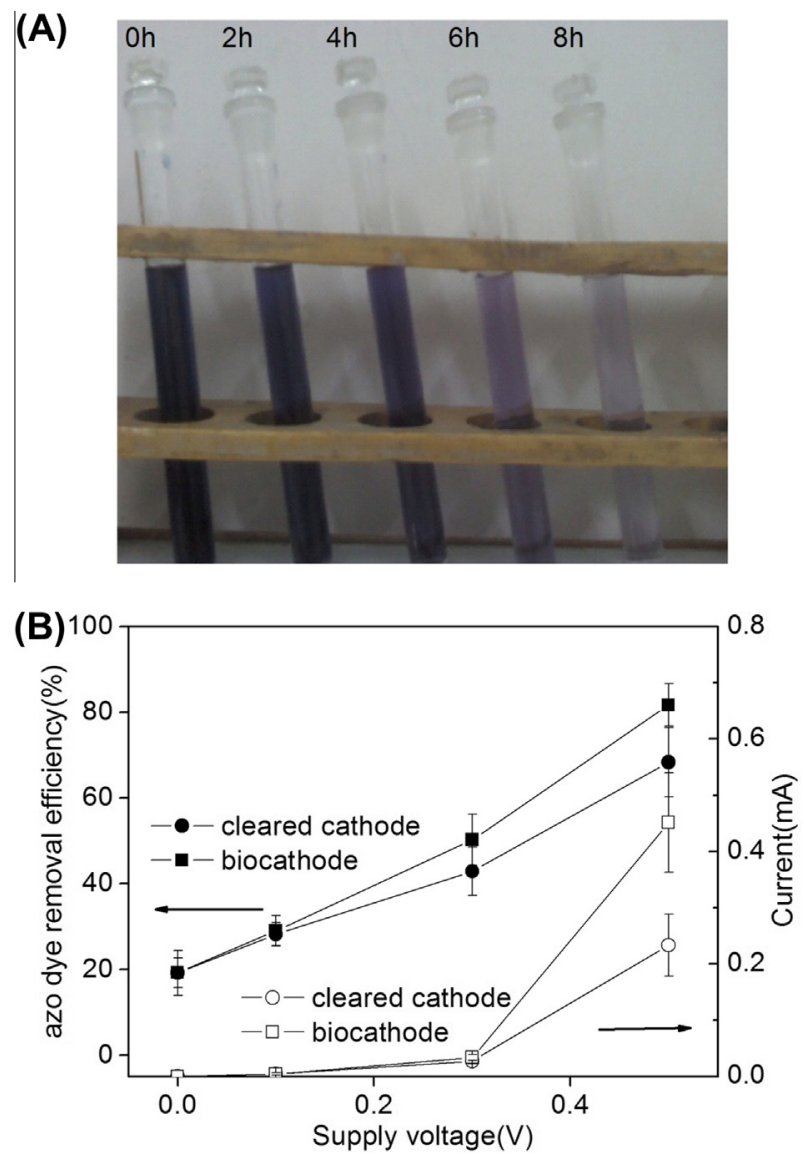

Fig. 3. Azo dye removal by BES with biocathode, (A) decoloration image of amido black 10B; (B) amido black 10B removal efficiency and current in BES with IEM. 


\section{Acknowledgements}

This research was supported by the National Natural Science Foundation of China (NSFC, Grant No. 51078100), by National Science Foundation for Distinguished Young Scholars (Grant No. 51225802), by Science Fund for Creative Research Groups of the National Natural Science Foundation of China (Grant No. 51121062), by the National High-tech R\&D Program of China (863 Program, Grant No. 2011AA060904), by The Ph.D. Programs Foundation of Ministry of Education of China (20102302110055), by "Hundred Talents Program" of the Chinese Academy of Sciences, and By State Key Laboratory of Urban Water Resource and Environment. HIT (Grant No. 2013DX02)

\section{Appendix A. Supplementary data}

Supplementary data associated with this article can be found, in the online version, at http://dx.doi.org/10.1016/j.biortech.2013. 07.082 .

\section{References}

Carbajosa, S., Malki, M., Caillard, R., Lopez, M.F., Javier Palomares, F., Martin Gago, J.A., Rodriguez, N., Amils, R., Fernandez, V.M., De Lacey, A.L., 2010 Electrochemical growth of Acidithiobacillus ferrooxidans on a graphite electrode for obtaining a biocathode for direct electrocatalytic reduction of oxygen. Biosens. Bioelectron. 26, 877-880.

Erable, B., Vandecandelaere, L., Faimali, M., Delia, M.L., Etcheverry, L., Vandamme, P. Bergel, A., 2010. Marine aerobic biofilm as biocathode catalyst. Bioelectrochemistry 78, 51-56.
He, Z., Mansfeld, F., 2009. Exploring the use of electrochemical impedance spectroscopy (EIS) in microbial fuel cell studies. Energy Environ. Sci. 2, 215-219.

Huang, L.P., Chen, J.W., Quan, X., Yang, F.L., 2010. Enhancement of hexavalent chromium reduction and electricity production from a biocathode microbial fuel cell. Bioresour. Technol. 33, 937-945.

Hong, B., Hu, Y.Y., Sun, J., 2012. Performance and microbial diversity of microbial fuel cells coupled with different cathode types during simultaneous azo dye decolorization and electricity generation. Bioresour. Technol. 111, 105-110.

Jeremiasse, A.W., Hamelers, H.V.M., Buisman, C.J.N., 2009. Microbial electrolysis cell with a microbial biocathode. Bioelectrochemistry 78, 29-43.

Logan, B.E., Hamelers, B., Rozendal, R., Schroder, U., Keller, J., Freguia, S., Aelterman, P., Verstraete, W., Rabaey, K., 2006. Microbial fuel cells: methodology and technology. Environ. Sci. Technol. 40, 5181-5192.

Liu, H., Hu, H.Q., Fan, Y.Z., 2008. Hydrogen production using single-chamber membrane-free microbial electrolysis cells. Water Res. 42, 4172-4178.

Mu, Y., Rabaey, K., Rozendal, R.A., Yuan, Z.G., Keller, J., 2009. Decolorization of azo dyes in bioelectrochemical systems. Environ. Sci. Technol. 43, 5137-5143.

Strycharz, S.M., Woodard, T.L., Johnson, J.P., Nevin, K.P., Sanford, R.A., Loffler, F.E., Lovley, D.R. 2008. Graphite electrode as a sole electron donor for reductive dechlorination of tetrachlorethene by Geobacter lovleyi. Appl. Environ. Microbiol. 74, 5943-5947.

Shen, J.Y., Feng, C.C., Zhang, Y.Y., Jia, F., Sun, X.Y., Li, J.S., Han, W.Q., Wang, L.J., Mu, Y., 2012. Bioelectrochemical system for recalcitrant $\rho$-nitrophenol removal. J. Hazard. Mater. 209, 516-519.

Wang, A.J., Sun, D., Cao, G.L., Cheng, H.Y., Ren, N.Q., Wu, W.M., Logan, B.E., 2011a. Integrated hydrogen production process from cellulose by combining dark fermentation, microbial fuel cells, and a microbial electrolysis cell. Bioresour. Technol. 102, 4137-4143.

Wang, A.J., Cheng, H.Y., Liang, B., Ren, N.Q., Cui, D., Lin, N., Kim, B.H., Rabaey, K. 2011b. Efficient reduction of nitrobenzene to aniline with a biocatalyzed cathode. Environ. Sci. Technol. 23, 10186-10193.

Wang, Y.Z., Wang, A.J., Liu, W.Z., Sun, Q., 2013. Enhanced azo dye removal through anode biofilmacclimation to toxicity in single-chamber biocatalyzed electrolysis system. Bioresour. Technol. 142, 688-692. 